\title{
Canabinoides na dor crónica: uma revisão baseada na evidência
}

Ricardo Garcia Silva, ${ }^{1}$ Sofia Piarali Remtula, ${ }^{1}$ Tiago Castelar Gonçalves ${ }^{1}$

\begin{abstract}
RESUMO
Objetivo: Avaliar a evidência existente relativa à eficácia de canabinoides no tratamento da dor crónica.

Fontes de dados: MEDLINE/PubMed, Cochrane Library, TRIP Database, National Guideline Clearing House, Canadian Medical Association Practice Guidelines.

Métodos de revisão: Utilizando os termos MeSH cannabis e chronic pain fez-se, em agosto de 2019, uma pesquisa de meta-análises (MA), revisões sistemáticas (RS), estudos observacionais (EO), ensaios clínicos (EC) e guidelines, publicados em português e inglês, sem limite temporal. Incluíram-se estudos realizados em adultos com dor crónica, independentemente da causa, submetidos a terapêutica com canabinoides, excluindo-se aqueles com intervenção em dor aguda. Utilizou-se a escala Strength of Recommendation Taxonomy, da American Academy of Family Physicians, para atribuir níveis de evidência (NE) e força de recomendação (FR).

Resultados: Dos 244 artigos encontrados, 16 cumpriram os critérios de inclusão: nove RS, quatro EC duplo-cegos aleatorizados e controlados com placebo, dois estudos retrospetivos de série de casos e um estudo prospetivo de série de casos. Todos os estudos selecionados abordavam dor crónica, mas de etiologia diversa (oncológica, neuropática, reumatológica, visceral). Os resultados entre os estudos não foram consistentes. Parece haver algum benefício na dor neuropática e na dor oncológica, embora haja consenso pelas revisões de que serão necessários estudos de maior dimensão e duração para que a utilização de canabinoides tenha evidência robusta. Podem verificar-se efeitos adversos gastrointestinais e nas funções cognitiva e motora, sobretudo com as preparações contendo maior dosagem de tetrahidrocanabinol. Não há evidência para utilização em dor de origem reumatológica ou visceral. Não se atribuiu NE 1 a qualquer estudo.

Conclusões: A utilização de canabinoides, embora promissora e com eventual benefício identificado em pequenos estudos para alguns tipos de dor crónica (sobretudo a neuropática), tem evidência limitada (FR B) e requer a realização de ensaios de maior qualidade e dimensão. Devem ser considerados a eficácia e os possíveis efeitos secundários a longo prazo em estudos de maior duração, algo que poderá ser alcançado com a crescente utilização dos fármacos na prática clínica. Com base na evidência disponível, os canabinoides poderão ser uma solução de última linha em casos de dor refratária neuropática e oncológica.
\end{abstract}

Palavras-chave: Canábis; Canabinoides; Dor crónica.

\section{INTRODUÇÃO}

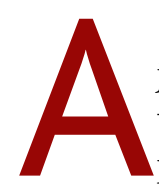
canábis (Cannabis sativa L) é uma planta cujos constituintes (sobretudo os canabinoides) têm vindo a ser associados a diversos efeitos psicoativos e medicinais. ${ }^{1-2}$ Já foram identificados mais de 100 canabinoides, ${ }^{1}$ presentes na planta sob a forma de ácidos. ${ }^{2}$ Os mais predominantes são, por ordem decrescente, o ácido tetrahidrocanabinóli-

1. Médico Interno de Medicina Geral e Familiar. USF Conde de Oeiras, ACeS Lisboa Ocidental e Oeiras. Oeiras, Portugal. co (ATHC), o ácido canabidiólico (ACBD), o ácido canabigerólico (ACBG) e o ácido canabicroménico (ACBC). Após aquecimento, estes ácidos são descarboxilados e originam as suas formas neutras, das quais as mais importantes e abundantes são o tetrahidrocanabinol (THC), responsável pelos efeitos psicoativos, e o canabidiol (CBD). ${ }^{2}$ Com o desenvolvimento de canabinoides sintéticos, como dronabinol e nabilona, e a descoberta de ligandos endógenos aos recetores canabinoides cerebrais, os originários na planta receberam a denominação de fitocanabinoides. ${ }^{3}$ 
O uso potencial destes compostos para efeitos terapêuticos tem motivado alterações da legislação em alguns países. ${ }^{4}$ Em Portugal, a Lei n. ${ }^{\circ} 33 / 2018$, de 18 de julho, estabeleceu o quadro legal para a utilização de medicamentos, preparações e substâncias à base da planta da canábis para fins medicinais, nomeadamente a sua prescrição e a sua dispensa em farmácia. ${ }^{5}$ Foi complementada pelo Decreto-Lei n. ${ }^{8} 8 / 2019$, de 15 de janeiro, o qual regulamenta as atividades de cultivo, extração e comerciais envolvendo estas preparações. ${ }^{6}$ No ano de 2019, o INFARMED publicou uma lista de condições para as quais o uso de canabinoides com intuito medicinal se encontra aprovado, com a ressalva de que estes devem ser ponderados apenas nos casos em que os tratamentos convencionais com medicamentos autorizados não produzam os efeitos esperados ou provoquem efeitos adversos relevantes. ${ }^{7}$ Nessa lista encontra-se a dor crónica (seja de origem oncológica ou neuropática), que se define como dor que recorre ou persiste por mais de três meses e se associa a impotência funcional ou perturbação emocional. ${ }^{8}$

O médico de família é, habitualmente, o primeiro ponto de contacto dos utentes com os serviços de saúde e estabelece com eles uma relação de continuidade e proximidade. Este facto contribui para que seja muitas vezes solicitado a esclarecer informação científica. Por outro lado, será porventura o clínico que mais se ocupa da gestão de dor crónica. Assim, perante estas recentes aprovações e o aumento do interesse público no tema, é necessário que detenha a melhor informação disponível nesta área. Com esta revisão pretende-se avaliar a evidência existente relativa à eficácia destas substâncias no tratamento da dor crónica.

\section{MÉTODOS}

Realizou-se, em agosto de 2019, uma pesquisa de meta-análises (MA), revisões sistemáticas (RS), estudos observacionais, ensaios clínicos e guidelines, publicados nas línguas portuguesa e inglesa, sem limite temporal, utilizando os termos MeSH cannabis e chronic pain nas bases de dados científicas MEDLINE/PubMed, The Cochrane Library, TRIP Database, National Guideline Clearing House e Canadian Medical Association Practice Guidelines.

Os critérios de elegibilidade foram os seguintes:

- População: indivíduos adultos com dor crónica, independentemente da causa;
- Intervenção: tratamento com substâncias derivadas da planta de canábis;

- Comparação: utilização de placebo ou tratamento com outros fármacos analgésicos;

- Outcome: melhoria sintomática, definida por diminuição da intensidade da dor.

Foram excluídos estudos que avaliassem a eficácia destas substâncias na dor aguda. Artigos de opinião e artigos de revisão clássica de tema foram também excluídos, à semelhança dos ensaios já incluídos em meta-análise ou revisão sistemática.

Utilizou-se a escala Strength of Recommendation Taxonomy (SORT), da American Academy of Family Physicians, para a avaliação dos níveis de evidência (NE) e da força de recomendação (FR).

\section{RESULTADOS}

Da pesquisa realizada resultou um total de $244 \mathrm{pu}-$ blicações, dos quais 16 cumpriam os critérios de inclusão: três RS com MA, cinco RS, uma revisão de RS, quatro ensaios clínicos aleatorizados e controlados (ECAC), dois estudos retrospetivos de série de casos e um estudo prospetivo de série de casos. O fluxograma representado na Figura 1 resume o processo de seleção dos estudos, que se encontram resumidos nas Tabelas 1, 2, 3, 4 e 5 .

Das três RS com MA (Tabela 1), duas procuraram avaliar eficácia e efeitos adversos de qualquer preparação contendo canabinoides em doentes com dor crónica de várias causas, comparativamente com placebo. ${ }^{9-10}$ Os resultados favorecem ligeiramente a utilização destes compostos, no que diz respeito a melhoria sintomática, com tamanho do efeito ( $\mathrm{g}$ de Hedges) de -0,61 $(-0,84$ a $-0,37)$ no trabalho de Martín-Sanchez e colaboradores $^{9}$ e de -0,40 (-0,58 a -0,21) na revisão de Aviram e colaboradores ${ }^{10}$ (NE 2). Aviram e colaboradores demonstraram que o efeito pode ser maior na dor neuropática e na dor oncológica, sobretudo pela via inalatória. No entanto, ambos os trabalhos fazem referência a efeitos adversos importantes, tanto nas funções cognitiva e motora ${ }^{9}$ como gastrointestinais ${ }^{10}$ (NE 2).

A RS com MA de Stockings e colaboradores estudou a eficácia de canabinoides em doentes com dor crónica não oncológica. ${ }^{11}$ Os ECAC incluídos na MA favorecem estas substâncias comparativamente com placebo para uma melhoria de $30 \%$ na intensidade da dor 


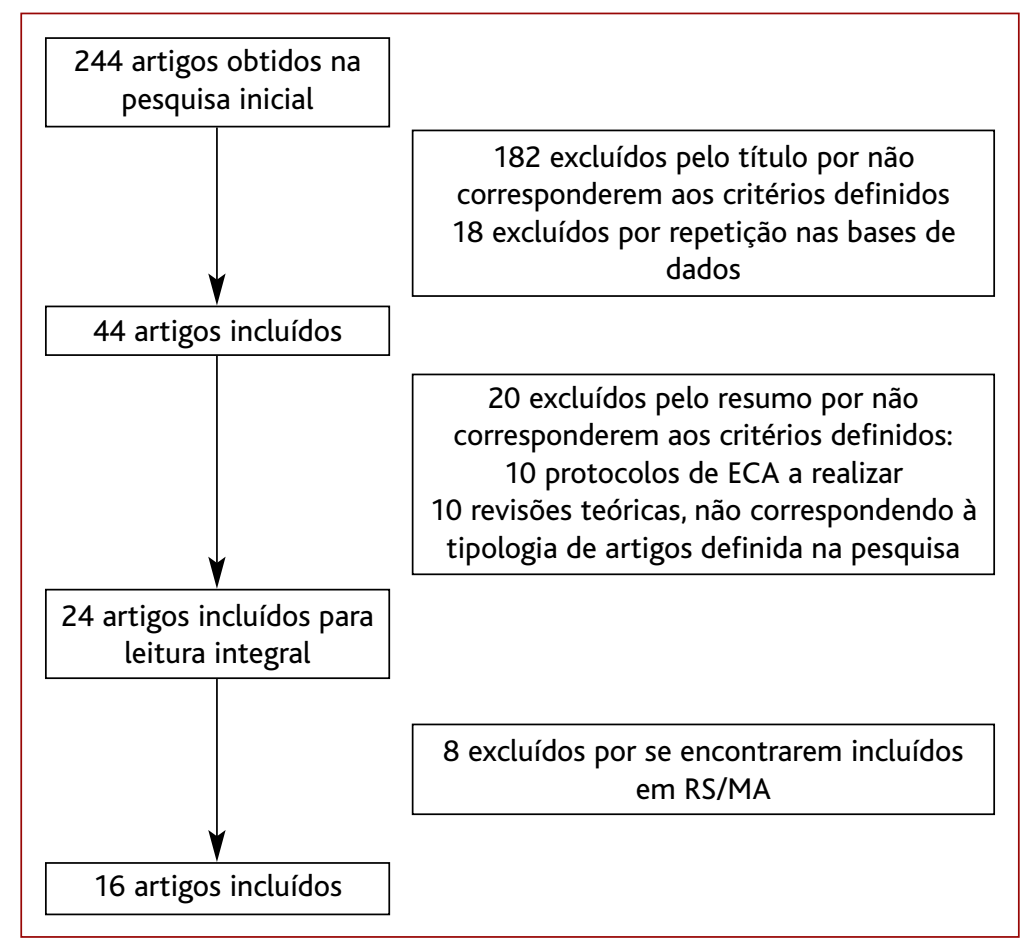

Figura 1. Fluxograma de seleção dos artigos.

Legenda: RS = Revisão sistemática; MA = Meta-análise.

(Odds Ratio $[\mathrm{OR}]=1,46$; IC95\% 1,16-1,84), embora não se tenha demonstrado diferença para os $50 \%(\mathrm{OR}=1,43$; IC95\% 0,97-2,11) (NE 2). Obteve-se, para 30\% de melhoria, um Number Needed to Treat (NNT) de 24, com Number Needed to Harm (NNH) de 6. Os estudos observacionais incluídos na RS não demonstraram evidência.

As RS com MA apresentaram várias limitações. Em todas verificou-se grande variabilidade entre os ensaios incluídos, não só no que respeita ao desenho dos mesmos, mas sobretudo à heterogeneidade de intervenções e etiologia da própria dor. A maioria dos ensaios incluídos tinha amostras de pequena dimensão e intervenções de curta duração. Como tal, o NE atribuído a estes estudos foi de 2. Na MA de Martín-Sanchez e colaboradores é também feita referência a ensaios com cruzamento com período washout curto. ${ }^{9}$ Stockings e colaboradores afirmam haver alto risco de viés na maioria dos ECAC incluídos. ${ }^{11}$

Das RS (sem MA) incluídas nesta revisão (Tabela 2), uma incidiu no tratamento de dor crónica não oncológica, ${ }^{12-14}$ três em dor neuropática ${ }^{13-15} \mathrm{e}$ uma em dor cró- nica, independentemente da causa, ${ }^{16}$ avaliando a eficácia de diferentes preparações e formas de administração dos canabinoides. Dos dezoito ensaios incluídos na revisão de Lynch e colaboradores, nove incidiram em dor neuropática, tendo demonstrado benefício comparando com placebo sobretudo nas vias inalada e sublingual/mucosa oral. ${ }^{12}$ Um destes estudos comparou ainda nabilona com hidrocodona; o efeito deste canabinoide na dor foi menor. Poderá haver melhoria das queixas álgicas de origem central em doentes com esclerose múltipla (dois em três ensaios mostraram eficácia). Nesta revisão foi incluído um artigo incidindo na dor em doentes com fibromialgia, que demonstrou melhoria com nabilona oral. O único ensaio que abordou a dor na artrite reumatoide obteve bons resultados com spray para a mucosa oral. Na globalidade verificou-se boa tolerância aos compostos e sem efeitos adversos graves (NE 2).

Deshpande e colaboradores incluíram na sua RS seis ECAC que demonstraram benefício estatisticamente significativo na utilização de fitocanabinoides inalados para tratamento da dor neuropática. ${ }^{13}$ No entanto, apenas três apresentaram benefício clinicamente relevante, definido por melhoria da intensidade da dor superior a $30 \%$. Após correção para os efeitos psicoativos dos canabinoides, dois ECAC mantiveram benefício demonstrado (NE 2). Não houve descrição de efeitos adversos graves.

Dos ensaios incluídos no artigo de Boychuk e colaboradores, apenas o que tinha maior amostra e que comparou nabiximols (spray para mucosa oral com fitocanabinoides THC e CBD) com placebo não demonstrou eficácia para dor neuropática. ${ }^{14}$ Os restantes fizeram-no, com seis ensaios a apresentar NNT variável entre 2 e 4 , parecendo haver maior benefício na utilização destas mesmas preparações em spray para a mucosa oral (NE 2). Os efeitos adversos (sobretudo cognitivos) foram mais frequentes nos ensaios que administraram canabinoides através da inalação de fumo da planta. Uma limitação importante deste trabalho é não ter havido critérios estabelecidos para definição de "diminuição de dor". 


\begin{tabular}{|c|c|c|c|c|}
\hline Referência (ano) & Tipo de estudo e amostra & Intervenção & Resultados & NE \\
\hline $\begin{array}{l}\text { Martín-Sánchez, } \\
\text { et al. (2009) }\end{array}$ & $\begin{array}{l}18 \text { Ensaios clínicos aleatorizados, } \\
\text { duplo-cego, controlados com } \\
\text { placebo, crossover } \\
n=809 \text { doentes } \\
\text { Duração de follow-up: } \\
4 \text { dias a } 6 \text { semanas }\end{array}$ & $\begin{array}{l}\text { Avaliar a eficácia e os malefícios } \\
\text { do tratamento da dor crónica } \\
\text { com canabinoides contendo THC } \\
\text { ou derivados sintéticos }\end{array}$ & $\begin{array}{l}\text { Discreta melhoria vs placebo } \\
\text { Hedge's g -0,61 (-0,84 a -0,37). } \\
\text { Número considerável de efeitos } \\
\text { adversos do sistema nervoso } \\
\text { central num curto período de } \\
\text { tempo: euforia (NNH 8); } \\
\text { alterações da perceção, } \\
\text { nomeadamente confusão, } \\
\text { desorientação, visão turva e } \\
\text { acufenos (NNH 7); alterações } \\
\text { motoras, nomeadamente ataxia, } \\
\text { disartria e fasciculações (NNH 5); } \\
\text { alterações da memória (NNH 8). }\end{array}$ & 2 \\
\hline $\begin{array}{l}\text { Aviram, et al. } \\
(2017)^{10}\end{array}$ & $\begin{array}{l}42 \text { Ensaios clínicos aleatorizados } \\
\text { ( } 24 \text { elegíveis para MA): } 27 \text { dor } \\
\text { neuropática, } 7 \text { dor oncológica } \\
n=2.437 \text { ( } 1.334 \text { na MA) } \\
\text { Duração de follow-up: } 2 \text { horas a } \\
12 \text { semanas }\end{array}$ & $\begin{array}{l}\text { Avaliar a eficácia e os efeitos } \\
\text { adversos dos canabinoides } \\
\text { naturais ou sintéticos no } \\
\text { tratamento da dor crónica }\end{array}$ & $\begin{array}{l}\text { Discreta melhoria vs placebo } \\
\text { Hedge's g }-0,40(-0,58 \text { a }-0,21 \text {, } \\
p<0,0001) \text {. } \\
\text { Dor neuropática: }-0,52 \text { Hedge's g } \\
(-0,75 \text { a }-0,30, p<0,0001) \text {. } \\
\text { Dor oncológica: }-0,76 \text { Hedge's g } \\
(-1,06 \text { to }-0,45, p<0,0001) \text {. } \\
\text { Heterogeneidade } \\
\text { estatisticamente significativa. } \\
\text { Os efeitos adversos mais } \\
\text { relatados foram os } \\
\text { gastrointestinais e do sistema } \\
\text { nervoso central. }\end{array}$ & 2 \\
\hline $\begin{array}{l}\text { Stockings, et al. } \\
(2018)^{11}\end{array}$ & $\begin{array}{l}57 \text { Estudos observacionais e } 47 \\
\text { ensaios clínicos aleatorizados } \\
\text { ( } 8 \text { elegíveis para MA): } \\
48 \text { dor neuropática; } \\
7 \text { fibromialgia; } \\
1 \text { artrite reumatóide } \\
\text { n=9958 ( } 1 \text { a } 649 ; \text { mediana: } 42 \text { ) } \\
\text { Follow-up variável ( } 1 \text { dia a } 8 \text { anos) }\end{array}$ & $\begin{array}{l}\text { Avaliar a eficácia dos canabinoides } \\
\text { naturais ou sintéticos no } \\
\text { tratamento da dor não oncológica }\end{array}$ & $\begin{array}{l}\text { Ensaios clínicos aleatorizados: } \\
\text { para redução de } 30 \% \text { de dor, } \\
\text { favorece canabinoides vs placebo } \\
\text { (OR=1,46; IC95\% 1,16-1,84), } \\
\text { NNT } 24 \text { (IC95\% 15-61); sem } \\
\text { diferença para 50\% (OR=1,43; } \\
\text { IC95\% 0,97-2,11); NNH } 6 \\
\text { (IC95\% 5-8). } \\
\text { Sem evidência nos estudos } \\
\text { observacionais. }\end{array}$ & 2 \\
\hline
\end{tabular}

Mucke e colaboradores avaliaram 16 ensaios clínicos aleatorizados, dos quais 10 utilizaram nabiximols. ${ }^{15}$
Compilando a informação de dez desses ensaios, os autores obtiveram valores de alívio da dor neuropática 
TABELA 2. Revisões sistemáticas

\begin{tabular}{|c|c|c|c|c|}
\hline Referência (ano) & Tipo de estudo e amostra & Intervenção & Resultados & NE \\
\hline $\begin{array}{l}\text { Lynch, et al. } \\
(2011)^{12}\end{array}$ & $\begin{array}{l}\text { Revisão sistemática de } 18 \\
\text { ensaios clínicos aleatorizados: } \\
9 \text { dor neuropática vs placebo; } \\
1 \text { dor associada a espasticidade } \\
\text { vs placebo; } 3 \text { dor central em } \\
\text { doentes com esclerose múltipla } \\
\text { vs placebo; } 1 \text { dor crónica de } \\
\text { origem espondilótica vs placebo; } \\
2 \text { dor não oncológica de diversas } \\
\text { etiologias ( } 1 \text { vs placebo, } 1 \text { vs } \\
\text { hidrocodona); } 1 \text { fibromialgia vs } \\
\text { placebo; } 1 \text { artrite reumatoide vs } \\
\text { placebo } \\
n=766 \text { doentes } \\
\text { Duração de follow-up: } 6 \text { horas a } \\
6 \text { semanas }\end{array}$ & $\begin{array}{l}\text { Avaliar a eficácia e segurança de } \\
\text { canabinoides com diversas } \\
\text { formas de administração no } \\
\text { tratamento da dor crónica } \\
\text { não-oncológica } \\
\text { Avaliar o nível de funcionalidade } \\
\text { com o uso dos canabinoides } \\
\text { Avaliar a tolerância dos efeitos } \\
\text { adversos dos canabinoides }\end{array}$ & $\begin{array}{l}\text { Dor neuropática: os } 9 \text { ensaios } \\
\text { ( } n=476) \text { mostraram melhoria da } \\
\text { dor. } \\
\text { Dor central na esclerose múltipla } \\
\text { ( } n=127): 2 \text { ( } n=90 \text { ) mostraram } \\
\text { benefício. } \\
\text { Dor não oncológica: melhoria vs } \\
\text { placebo ( } n=60) \text {, inferior a } \\
\text { hidrocodona ( } n=30) \text {. } \\
\text { Dor associada a espasticidade } \\
\text { ( } n=13 \text { ): benefício. } \\
\text { Dor espondilótica ( } n=30 \text { ): } \\
\text { benefício. } \\
\text { Artrite reumatoide ( } n=58 \text { ): } \\
\text { benefício. } \\
\text { Fibromialgia ( } n=40 \text { ): benefício. } \\
\text { Melhoria significativa da } \\
\text { qualidade do sono. } \\
\text { Os efeitos adversos mais comuns } \\
\text { (sedação, tonturas, boca seca, } \\
\text { náusea e dificuldade de } \\
\text { concentração) foram na } \\
\text { generalidade bem tolerados. }\end{array}$ & 2 \\
\hline $\begin{array}{l}\text { Deshpande, et al. } \\
(2015)^{13}\end{array}$ & $\begin{array}{l}\text { Revisão sistemática de } 6 \text { ensaios } \\
\text { clínicos aleatorizados em doentes } \\
\text { com dor não oncológica: } \\
5 \text { ensaios duplo-cego controlados } \\
\text { com placebo, crossover; } 1 \text { ensaio } \\
\text { controlado com placebo } \\
n=226 \\
\text { Duração de follow-up: } 3 \text { horas } \\
\text { a } 8 \text { semanas }\end{array}$ & $\begin{array}{l}\text { Avaliar a eficácia dos } \\
\text { canabinoides fumados ou } \\
\text { vaporizados no tratamento da } \\
\text { dor não oncológica } \\
\text { Avaliar o efeito dos canabinoides } \\
\text { fumados ou vaporizados a nível } \\
\text { da funcionalidade, identificar } \\
\text { doses terapêuticas e efeitos } \\
\text { adversos }\end{array}$ & $\begin{array}{l}\text { Todos os estudos demonstraram } \\
\text { benefício estatisticamente } \\
\text { significativo no alívio da dor } \\
\text { com baixas doses; } 3 \text { estudos } \\
\text { mostraram benefício } \\
\text { clinicamente relevante. } \\
\text { Após correção para efeitos } \\
\text { psicoativos de THC, apenas } 2 \\
\text { ( } n=67) \text { estudos mostraram } \\
\text { benefício. } \\
\text { A curto prazo foi bem tolerado, } \\
\text { mas os efeitos psicoativos e } \\
\text { neurocognitivos a longo prazo } \\
\text { permanecem desconhecidos. }\end{array}$ & 2 \\
\hline
\end{tabular}

superior a $30 \%$ em $39 \%$ dos doentes sob canabinoides, comparando com $33 \%$ daqueles a quem se atribuiu pla- cebo, chegando a um NNT de 11 (IC95\% 7-33). Para $50 \%$ de diminuição da intensidade da dor oito estudos 


\begin{tabular}{|c|c|c|c|c|}
\hline Referência (ano) & Tipo de estudo e amostra & Intervenção & Resultados & NE \\
\hline $\begin{array}{l}\text { Boychuk, et al. } \\
(2015)^{14}\end{array}$ & $\begin{array}{l}13 \text { Ensaios clínicos aleatorizados: } \\
\text { todos em doentes com dor } \\
\text { neuropática, comparando com } \\
\text { placebo } \\
n=771 \\
\text { Duração de follow-up: } 1 \text { a } 6 \\
\text { semanas }\end{array}$ & $\begin{array}{l}\text { Avaliar a eficácia dos } \\
\text { canabinoides naturais ou } \\
\text { sintéticos no tratamento da dor } \\
\text { não oncológica }\end{array}$ & $\begin{array}{l}\text { Em } 6 \text { estudos ( } n=255) \text { o NNT } \\
\text { foi de } 2 \text {-4. } \\
\text { O maior estudo do grupo } \\
\text { ( } n=339 \text { ) não mostrou benefício. } \\
\text { Os restantes } 12 \text { estudos } \\
\text { sugerem redução significativa da } \\
\text { dor comparando com placebo. } \\
\text { Os sprays para a mucosa oral } \\
\text { parecem mais eficazes e mais } \\
\text { seguros. }\end{array}$ & 2 \\
\hline $\begin{array}{l}\text { Mucke, et al. } \\
(2018)^{15}\end{array}$ & $\begin{array}{l}16 \text { Ensaios clínicos aleatorizados: } \\
\text { todos em doentes com dor } \\
\text { neuropática } \\
n=1.750 \\
\begin{array}{l}\text { Duração de follow-up: } 2 \text { a } 26 \\
\text { semanas }\end{array}\end{array}$ & $\begin{array}{l}\text { Avaliar a eficácia dos } \\
\text { canabinoides naturais ou } \\
\text { sintéticos no tratamento da dor } \\
\text { crónica neuropática }\end{array}$ & $\begin{array}{l}\text { Em } 10 \text { estudos ( } n=819):>30 \% \\
\text { de alívio em } 39 \% \text { vs } 33 \% \text { com } \\
\text { placebo; NNT=11 (IC95\% } 7 \text { a 33). } \\
\text { Em } 8 \text { estudos ( } n=526):>50 \% \\
\text { alívio em } 21 \% \text { vs } 17 \% \text { com } \\
\text { placebo; NNT=20 (IC95\% } 11 \text { a } \\
\text { 100). } \\
\text { Em } 13 \text { estudos ( } n=989) \text { : } \\
\text { NNH=25 (IC95\% } 16 \text { a } 50) \text {. } \\
\text { Evidência de baixa qualidade, } \\
\text { segundo os autores. }\end{array}$ & 2 \\
\hline $\begin{array}{l}\text { Campbell, et al. } \\
(2001)^{16}\end{array}$ & $\begin{array}{l}\text { Revisão sistemática de } 9 \text { ensaios } \\
\text { clínicos aleatorizados: } 5 \text { dor } \\
\text { crónica oncológica; } 2 \text { dor crónica } \\
\text { não-oncológica; } 2 \text { dor aguda } \\
\text { pós-operatória } \\
n=202 \text { doentes } \\
\text { Duração de follow-up: } 6 \text { horas a } \\
5 \text { meses }\end{array}$ & $\begin{array}{l}\text { Avaliar se o tratamento da dor } \\
\text { com canabinoides em várias } \\
\text { formas de administração é } \\
\text { efetivo e seguro }\end{array}$ & $\begin{array}{l}\text { Dor oncológica ( } n=128) \text { : } \\
2 \text { estudos vs placebo com } \\
\text { melhoria; } 1 \text { estudo vs placebo e } \\
\text { codeína sem melhoria; } 2 \text { estudos } \\
\text { vs placebo e codeína com } \\
\text { melhoria em relação a placebo, } \\
\text { mas não superior a codeína. } \\
\text { Dor não oncológica ( } n=2) \text { : } \\
1 \text { estudo sem melhoria vs } \\
\text { placebo em doente com febre } \\
\text { mediterrânica familiar; } 1 \text { estudo } \\
\text { com benefício comparável a } \\
\text { codeína em doente com dor } \\
\text { neuropática }\end{array}$ & 2 \\
\hline
\end{tabular}

continham informação, tendo-se atingido esses parâmetros em $21 \%$ dos doentes no braço terapêutico con- tra $17 \%$ no braço sob placebo, NNT de 20 (IC95\% 11-100) (NE 2). Não se verificaram efeitos adversos 
TABELA 3. Revisão de revisões sistemáticas

\begin{tabular}{|c|c|c|c|c|}
\hline Referência (ano) & Tipo de estudo e amostra & Intervenção & Resultados & NE \\
\hline $\begin{array}{l}\text { Hauser, et al. } \\
(2017)^{17}\end{array}$ & $\begin{array}{l}10 \text { Revisões sistemáticas de } \\
\text { ensaios clínicos aleatorizados: } \\
6 \text { com dados sobre dor } \\
\text { neuropática; } 3 \text { dor de doenças } \\
\text { reumáticas; } 2 \text { dor oncológica } \\
\text { Follow-up variável dos ensaios } \\
\text { incluídos nas revisões: } 4 \text { horas } \\
\text { a } 15 \text { semanas }\end{array}$ & $\begin{array}{l}\text { Avaliar a eficácia, aceitabilidade } \\
\text { e segurança dos canabinoides } \\
\text { naturais e sintéticos nos } \\
\text { doentes com dor crónica } \\
\text { (oncológica ou não } \\
\text { oncológica) }\end{array}$ & $\begin{array}{l}\text { Resultados inconsistentes na } \\
\text { dor neuropática. } \\
\text { Sem evidência de eficácia } \\
\text { superior a placebo na dor } \\
\text { reumática. } \\
\text { Não superior a placebo para } \\
\text { dor oncológica. } \\
\text { Resultados inconsistentes na } \\
\text { tolerância e segurança. }\end{array}$ & $\begin{array}{l}\text { Não } \\
\text { atribuído }\end{array}$ \\
\hline
\end{tabular}

clinicamente relevantes, tendo sido obtido um NNH de 25, com base em dados de 13 artigos. Os próprios autores classificam os estudos incluídos como de baixa qualidade.

A RS de Campbell e colaboradores, ao incluir estudos realizados em indivíduos com dor crónica, independentemente da causa, avaliou cinco ensaios clínicos realizados em doentes com dor de origem oncológica e dois em casos de dor não oncológica ${ }^{16}$ Em relação à dor oncológica, os autores verificaram que um estudo não demonstrou melhoria sintomática, enquanto dois outros demonstraram superioridade em relação a placebo, mas não quando comparado com codeína (NE 2). Os artigos com resultados favoráveis utilizaram preparações de THC oral em doses variáveis (5-20mg), com maior incidência de efeitos adversos (sobretudo sedação e alterações cognitivas) nas dosagens superiores a 10mg. Na dor não oncológica, um artigo não demonstrou superioridade em relação a placebo, enquanto outro mostrou benefício superior a placebo e comparável a codeína. Em ambos foram utilizadas formulações de THC oral. Realça-se que ambos correspondem a estudos com cruzamento, mas apenas com um doente em cada estudo, o que não permite formular conclusões. Como sucedeu no trabalho de Boychuk e colaboradores, os critérios para definição de alívio de dor não foram especificados.

Como verificado nas RS com MA, às RS incluídas neste estudo atribuiu-se NE 2. As limitações das RS são se- melhantes às explicitadas para as RS com MA, nomeadamente no que respeita ao tamanho das amostras dos ensaios e heterogeneidade das intervenções e de etiologias.

O trabalho de Hauser e colaboradores ${ }^{17}$ corresponde a uma revisão de RS (Tabela 3), uma metodologia recente que agrega a informação de diferentes RS que abordem o mesmo tópico. ${ }^{18}$ Foram incluídas 10 RS de ensaios clínicos aleatorizados, das quais seis abordaram a dor neuropática, três a dor de origem reumatológica e duas a dor oncológica. Nos ensaios clínicos incluídos em cada RS foram utilizadas diferentes substâncias, formas de administração e dosagens de canabinoides. Relativamente à dor neuropática, os resultados foram inconsistentes entre as RS, não permitindo inferir a eficácia. Não houve eficácia superior a placebo, tanto na dor de origem reumatológica como na oncológica. A este trabalho não foi atribuído nível de evidência dado tratar-se de uma metodologia não contemplada nos critérios da escala SORT.

Relativamente aos ECAC (Tabela 4), todos os incluídos na revisão foram duplamente cegos. De Vries e colaboradores investigaram a eficácia de comprimidos de THC para a dor abdominal crónica em contexto pós-cirúrgico ou de pancreatite crónica, não tendo obtido melhoria estatisticamente significativa comparativamente com placebo (NE 2). ${ }^{19}$

O ECAC com cruzamento de Weizman e colaboradores incidiu em doentes com dor crónica neuropática radicular, avaliando a eficácia de THC sublingual. ${ }^{20} \mathrm{Ve}-$ 


\begin{tabular}{|c|c|c|c|c|}
\hline Referência (ano) & Tipo de estudo e amostra & Intervenção & Resultados & NE \\
\hline $\begin{array}{l}\text { De Vries, et al. } \\
(2017)^{19}\end{array}$ & $\begin{array}{l}\text { Estudo fase II. } \\
65 \text { doentes com dor abdominal } \\
\text { crónica (contexto de pós cirurgia } \\
\text { ou pancreatite crónica), escala } \\
\text { numérica } \geq 3 \text {. } \\
\text { Duração de follow-up: } 52 \text { dias. }\end{array}$ & $\begin{array}{l}\text { Avaliar a eficácia analgésica, } \\
\text { farmacocinética, segurança e } \\
\text { tolerabilidade de um comprimido } \\
\text { oral contendo THC purificado em } \\
\text { doentes com dor abdominal } \\
\text { crónica }\end{array}$ & $\begin{array}{l}\text { Redução média da dor em escala } \\
\text { visual análoga no braço sob THC } \\
\text { de } 40 \%, 37 \% \text { no braço sob } \\
\text { placebo ( } p=0,901) \text {. } \\
\text { Sem significado estatístico. }\end{array}$ & 2 \\
\hline $\begin{array}{l}\text { Weizman, et al. } \\
(2018)^{20}\end{array}$ & $\begin{array}{l}\text { Estudo aleatorizado, duplo-cego, } \\
\text { controlado por placebo. Amostra } \\
\text { de } 15 \text { doentes do sexo masculino } \\
\text { com dor crónica neuropática } \\
\text { radicular > } 6 \text { meses com > } 40 \\
\text { pontos numa escala analógica } \\
\text { até } 100 \text { (VAS). } \\
\text { Toma única, com avaliação } 1 \text { e } \\
2 \text { horas após administração. }\end{array}$ & $\begin{array}{l}\text { Caracterizar as alterações } \\
\text { cerebrais funcionais envolvidas } \\
\text { na modulação de } \delta \text {-9-tetra- } \\
\text {-hidrocanabinol (THC) da dor } \\
\text { neuropática crónica }\end{array}$ & $\begin{array}{l}\text { Redução estatisticamente } \\
\text { significativa da dor avaliada pelo } \\
\text { VAS com THC sublingual } \\
\text { (diminuição com THC } 18,8 \pm 5,6 \text {, } \\
\text { placebo } 8,7 \pm 5,5, p<0,05 \text { ). }\end{array}$ & 2 \\
\hline $\begin{array}{l}\text { Lichtman, et al. } \\
(2018)^{21}\end{array}$ & $\begin{array}{l}\text { Estudo de fase } 3 \text {, duplo-cego, } \\
\text { aleatorizado, controlado por } \\
\text { placebo. Doentes com dor crónica } \\
\text { oncológica, escala numérica } \geq 4 \\
\text { e } \leq 8 \text { com terapêutica otimizada } \\
\text { com opiáceos. } \\
\text { Duração de follow-up: } 5 \text { semanas } \\
n=291\end{array}$ & $\begin{array}{l}\text { Avaliar nabiximols adjuvantes } \\
\left.\text { (Sativex }{ }^{\circledR}\right) \text {, um extrato de } \\
\text { Cannabis sativa contendo } \Delta 9 \text { - } \\
\text {-tetra-hidrocanabinol ( } 27 \mathrm{mg} / \mathrm{mL}) \\
\text { e canabidiol }(25 \mathrm{mg} / \mathrm{mL}), \text { em } \\
\text { doentes com cancro em estádios } \\
\text { avançados, com dor crónica não } \\
\text { controlada pela terapêutica } \\
\text { opioide. }\end{array}$ & $\begin{array}{l}\text { Redução de } 10,7 \% \text { no grupo de } \\
\text { canabinoides vs } 4,5 \% \text { no grupo } \\
\text { placebo ( } p=0,0854) \text {. } \\
\text { Superioridade dos canabinoides } \\
\text { em todos os instrumentos de } \\
\text { qualidade de vida. }\end{array}$ & 2 \\
\hline $\begin{array}{l}\text { Van de Donk, et } \\
\text { al. }(2019)^{22}\end{array}$ & $\begin{array}{l}\text { Estudo cruzado de } 4 \text { vias, } \\
\text { controlado por placebo, } \\
\text { aleatorizado. Avaliados os efeitos } \\
\text { analgésicos de } 4 \text { tipos de } \\
\text { cannabis inalada com nível } \\
\text { terapêutico em } 20 \text { doentes com } \\
\text { dor crónica com fibromialgia } 1 \text {, } \\
2 \text { e } 3 \text { horas após administração. }\end{array}$ & $\begin{array}{l}\text { Avaliação dos efeitos da cannabis } \\
\text { no alívio da dor em relação ao } \\
\text { placebo de cannabis com cheiro, } \\
\text { aparência e manuseio } \\
\text { semelhantes aos de outras } \\
\text { variedades. Avaliação do alívio da } \\
\text { dor por pressão, dor elétrica e } \\
\text { dor espontânea (endpoints } \\
\text { primários), bem como os efeitos } \\
\text { subjetivos e psicotrópicos. }\end{array}$ & $\begin{array}{l}\text { Nenhuma preparação } \\
\text { demonstrou superioridade na } \\
\text { redução da dor vs placebo (VAS } \\
\text { e teste por estímulo elétrico). } \\
\text { Preparações com THC em doses } \\
\text { altas aumentaram a tolerância à } \\
\text { pressão }(p<0,001) \text {. }\end{array}$ & 2 \\
\hline
\end{tabular}

rificou-se diminuição estatisticamente significativa da dor avaliada em escalas visuais com o THC sublingual (NE 2). No entanto, este estudo teve uma amostra de apenas 15 doentes, todos do sexo masculino e com avaliação em toma única.

Lichtman e colaboradores avaliaram a utilização de spray para a mucosa oral com THC e CBD na proporção de 1:1 em doentes com dor crónica de origem on- cológica, já sob analgesia com opiáceos, ao longo de cinco semanas. ${ }^{21}$ Apesar de se ter verificado benefício através dos instrumentos de avaliação de qualidade de vida e ligeira melhoria das queixas álgicas no braço sob tratamento, esta não foi estatisticamente significativa $(p=0,0854)$ (NE 2). Verificou-se heterogeneidade tanto no que respeita à origem da neoplasia como no tipo e dose de opiáceo realizado pelos doentes. 


\begin{tabular}{|c|c|c|c|c|}
\hline Referência (ano) & Tipo de estudo e amostra & Intervenção & Resultados & NE \\
\hline $\begin{array}{l}\text { Bellnier, et al. } \\
(2017)^{23}\end{array}$ & $\begin{array}{l}\text { Estudo retrospetivo de série de } \\
\text { casos. } \\
29 \text { doentes com dor crónica de } \\
\text { várias causas (incluindo } \\
\text { oncológica). }\end{array}$ & $\begin{array}{l}\text { Avaliação da eficácia de canábis } \\
\text { medicinal em doentes com dor } \\
\text { crónica de várias causas } \\
\text { (incluindo oncológica), } \\
\text { comparando o European Quality } \\
\text { of Life } 5 \text { Dimension Questionnaire } \\
\text { (EQ-5D) e a Pain Quality } \\
\text { Assessment Scale (PQAS) basal e } \\
3 \text { meses após terapêutica. }\end{array}$ & $\begin{array}{l}\text { Melhoria do EQ-5D de } \\
36,08 \pm 19,85 \text { para } 64,43 \pm 19,15 \\
(p<0,0001) \text {. } \\
\text { Diminuição da dor de } 6,76 \text { para } \\
2,04 \text { ( } p<0,0001) \text {. }\end{array}$ & 3 \\
\hline $\begin{array}{l}\text { Fanelli, et al. } \\
(2017)^{24}\end{array}$ & $\begin{array}{l}\text { Estudo retrospetivo de série de } \\
\text { casos. } \\
614 \text { doentes com dor crónica } \\
\text { refratária de várias causas } \\
\text { (incluindo oncológica). }\end{array}$ & $\begin{array}{l}\text { Avaliação da eficácia via } \\
\text { feedback dos doentes sob } \\
\text { tratamento com infusão de } \\
\text { canábis ou vaporizada. }\end{array}$ & $\begin{array}{l}76,2 \% \text { dos doentes não } \\
\text { descontinuaram a terapêutica } \\
\text { até à } 1^{\text {a }} \text { consulta (em média, } \\
98,42 \text { dias depois), dos quais } \\
64,7 \% \text { referiram melhoria } \\
\text { sintomática. } 23.8 \% \\
\text { descontinuaram ( } 3,7 \% \text { por } \\
\text { agravamento, } 61,7 \% \text { por efeitos } \\
\text { adversos não graves). }\end{array}$ & 3 \\
\hline $\begin{array}{l}\text { Crowley, et al. } \\
(2018)^{25}\end{array}$ & $\begin{array}{l}\text { Estudo prospetivo de série de } \\
\text { casos, sem controlo, sem blinding } \\
\text { com } 49 \text { doentes com dor crónica } \\
\text { não oncológica. }\end{array}$ & $\begin{array}{l}\text { Avaliação da dor via escala } \\
\text { numérica } 0-10 \text { após intervalo } \\
\text { variável 1-12 semanas sob } \\
\text { tratamento com THC e CBD em } \\
\text { doses e proporções variáveis, } \\
\text { orodispersível. }\end{array}$ & $\begin{array}{l}\text { Redução em } 4,9 \pm 2 \text { pontos }- \text { de } \\
7,4 \pm 1,3 \text { para } 2,4 \pm 1,8(p<0,001)\end{array}$ & 3 \\
\hline
\end{tabular}

No trabalho de Van de Donk e colaboradores, um estudo com cruzamento, os autores investigaram os efeitos de diferentes preparações, contendo concentrações diferentes de THC e CBD, em mulheres com fibromialgia. ${ }^{22}$ Nenhuma preparação mostrou superioridade na redução da dor comparativamente com o placebo (NE 2).

Os dois estudos retrospetivos de série de casos incluídos nesta revisão (Tabela 5) utilizaram amostras de doentes com dor crónica de diferentes etiologias, nos quais foram utilizados canabinoides em diferentes proporções e com diferentes formas de administração, excluindo a via inalatória. ${ }^{23-24}$ Bellnier e colaboradores obtiveram resultados de diminuição de dor de média de 6,76 para 2,04 em escala de avaliação da dor $(p<0,0001)$ (NE 3) ${ }^{23}$ No estudo de Fanelli e colaboradores, os doentes foram submetidos a tratamento com canabinoides de administração oral com concentrações variáveis de
THC e CBD. ${ }^{24}$ Dos $76,2 \%$ dos doentes que não descontinuaram a terapêutica até à primeira consulta de reavaliação, $64,7 \%$ referiram melhoria sintomática, não tendo sido utilizada nenhuma escala validada. Dos $23,8 \%$ que descontinuaram, $3,7 \%$ fizeram-no por agravamento das queixas e $61,7 \%$ por efeitos adversos não graves (NE 3).

O único estudo prospetivo incluído nesta revisão (Tabela 5) foi de Crowley e colaboradores, de uma série de casos, realizado em doentes com dor crónica não oncológica. ${ }^{25}$ Foram utilizados tratamentos com preparações orodispersíveis contendo CBD e THC em doses variáveis. Os autores obtiveram melhoria das queixas álgicas estatisticamente significativa $(p<0,001)(\mathrm{NE} 3)$.

\section{CONCLUSÕES}

De acordo com a literatura disponível atualmente, esta revisão permite concluir que a evidência para a 
utilização de canabinoides na dor crónica é limitada (FR B), com maior benefício potencial nos casos de dor crónica de origem neuropática e oncológica, para os quais há mais estudos realizados. A evidência atual não é suficiente para demonstrar benefício para a dor crónica por patologia do foro reumatológico (FR B).

No que respeita à dor neuropática, a maioria dos estudos incluídos demonstrou benefício desta terapêutica, destacando-se uma RS com MA, ${ }^{10}$ três RS ${ }^{12-13,15}$ e o ECA de Weizman e colaboradores. ${ }^{20}$ No entanto, para além das limitações já referidas para estes estudos, destaca-se o facto de o NNT para doentes com dor crónica não oncológica, calculado em duas destas RS com MA, ${ }^{11,15}$ variar entre 11 e 24 . Estes valores são elevados, sobretudo quando comparados com os NNT para outros fármacos utilizados em dor crónica neuropática, como o caso da pregabalina (NNT 7,7), gabapentina (NNT 7,2), tramadol (NNT 4,7) e amitriptilina (NNT $3,6){ }^{26}$

Na dor crónica oncológica, a RS com MA de Aviram e colaboradores concluiu haver ligeiro benefício. ${ }^{10} \mathrm{O}$ ECA de Lichtman e colaboradores não demonstrou alívio estatisticamente significativo da dor, mas melhoria na sensação de bem-estar e qualidade de vida dos doentes, potencial variável de confusão em alguns estudos. $^{21}$

Dos artigos que abordaram a dor crónica de origem reumática, apenas os incluídos na revisão sistemática de Lynch e colaboradores mostraram diminuição das queixas álgicas. ${ }^{12}$ Dos restantes, nenhum demonstrou evidência para a utilização de canabinoides. ${ }^{17,22}$

A revisão de revisões sistemáticas não concluiu benefício para dor crónica de qualquer etiologia. ${ }^{17}$

Relativamente a efeitos adversos, a incidência destes parece estar relacionada com formulações com maior concentração de THC relativamente a CBD, tendo-se verificado heterogeneidade entre os estudos no que respeita às formulações utilizadas. ${ }^{24} \mathrm{O} \mathrm{NNH}$ obtido no trabalho de Stockings e colaboradores ${ }^{11}$ foi de seis, valor inferior ao de outros fármacos, como a pregabalina (NNH 13,9). ${ }^{26}$

A dor crónica, pelo impacto que apresenta na qualidade de vida dos doentes e, muitas vezes, pelo seu caráter incurável, constitui um desafio para os clínicos. $\mathrm{O}$ aparecimento de novos fármacos permite alargar o espectro da terapêutica a utilizar. No caso das formula- ções contendo canabinoides são necessários mais estudos, com amostras de maiores dimensões e mais homogéneas, por forma a que estas possam vir a ser consideradas opções terapêuticas que não de última linha. A forma de administração (oral/inalada/sublingual) e a concentração e proporção dos diferentes constituintes dos fármacos variam consideravelmente de estudo para estudo, resultando em evidência díspar e perfil de efeitos adversos variável, sendo também necessários ensaios de maior duração para avaliar a segurança destes fármacos a médio e longo prazo.

Com esta revisão, a prática do médico de família na abordagem da dor não sofrerá alterações. No entanto, este tema é atual e tem despertado crescente interesse de domínio público. Reforça-se a necessidade de o médico de família, como primeiro contacto do utente com o serviço de saúde, estar informado e facultar respostas tão cientificamente comprovadas quanto possível.

\section{AGRADECIMENTOS}

Os autores expressam publicamente o seu agradecimento aos especialistas em medicina geral e familiar, Dra. Maria João Martins e Dr. Carlos Silva Russo, médicos de família na USF Conde de Oeiras, pelo apoio prestado ao longo da realização deste trabalho.

\section{REFERÊNCIAS BIBLIOGRÁFICAS}

1. Pollastro F, Minassi A, Fresu LG. Cannabis phenolics and their bioactivities. Curr Med Chem. 2018;25(10):1160-85.

2. Andre CM, Hausman JF, Guerriero G. Cannabis sativa: the plant of the thousand and one molecules. Front Plant Sci. 2016;7:19.

3. ElSohly MA, Slade D. Chemical constituents of marijuana: the complex mixture of natural cannabinoids. Life Sci. 2005;78(5):539-48.

4. Abuhasira R, Shbiro L, Landschaft Y. Medical use of cannabis and cannabinoids containing products: regulations in Europe and North America. Eur J Intern Med. 2018;49:2-6.

5. Lei . $^{\circ}$ 33/2018, de 18 de julho. Diário da República. $1^{\text {a }}$ série(137).

6. Decreto-Lei n. ${ }^{\circ} 8 / 2019$, de 15 de janeiro. Diário da República. $1^{\text {a }}$ série(10).

7. INFARMED. Deliberação n. ${ }^{\circ}$ 11/CD/2019. Lisboa: INFARMED; 2019.

8. Treede RD, Rief W, Barke A, Aziz Q, Bennett MI, Benoliel R, et al. Chronic pain as a symptom or a disease: the IASP Classification of Chronic Pain for the International Classification of Diseases (ICD-11). Pain. 2019;160(1):19-27.

9. Martín-Sánchez E, Furukawa TA, Taylor J, Martin JL. Systematic review and meta-analysis of cannabis treatment for chronic pain. Pain Med. 2009;10(8):1353-68.

10. Aviram J, Samuelly-Leichtag G. Efficacy of cannabis-based medicines for pain management: a systematic review and meta-analysis of randomized controlled trials. Pain Physician. 2017;20(6):E755-96.

11. Stockings E, Campbell G, Hall WD, Nielsen S, Zagic D, Rahman R, et al. Cannabis and cannabinoids for the treatment of people with chronic 
noncancer pain conditions: a systematic review and meta-analysis of controlled and observational studies. Pain. 2018;159(10):1932-54.

12. Lynch ME, Campbell F. Cannabinoids for treatment of chronic non-cancer pain: a systematic review of randomized trials. $\mathrm{Br} J \mathrm{Clin}$ Pharmacol. 2011;72(5):735-44.

13. Deshpande A, Mailis-Gagnon A, Zoheiry N, Lakha SF. Efficacy and adverse effects of medical marijuana for chronic noncancer pain: systematic review of randomized controlled trials. Can Fam Physician. 2015;61(8):e372-81.

14. Boychuk DG, Goddard G, Mauro G, Orellana MF. The effectiveness of cannabinoids in the management of chronic nonmalignant neuropathic pain: a systematic review. J Oral Facial Pain Headache. 2015;29(1):7-14.

15. Mücke M, Phillips T, Radbruch L, Petzke F, Häuser W. Cannabis-based medicines for chronic neuropathic pain in adults. Cochrane Database Syst Rev. 2018;3(3):CD012182.

16. Campbell FA, Tramèr MR, Carroll D, Reynolds DJ, Moore RA, McQuay $H J$. Are cannabinoids an effective and safe treatment option in the management of pain? A qualitative systematic review. BMJ. 2001;323(7303):13-6.

17. Häuser W, Petzke F, Fitzcharles MA. Efficacy, tolerability and safety of cannabis-based medicines for chronic pain management: an overview of systematic reviews. Eur J Pain. 2018;22(3):455-70.

18. Hunt H, Pollock A, Campbell P, Estcourt L, Brunton G. An introduction to overviews of reviews: planning a relevant research question and objective for an overview. Syst Rev. 2018;7(1):39.

19. de Vries M, van Rijckevorsel DC, Vissers KC, Wilder-Smith OH, van Goor $H$. Tetrahydrocannabinol does not reduce pain in patients with chronic abdominal pain in a phase 2 placebo-controlled study. Clin Gastroenterol Hepatol. 2017;15(7):1079-86.e4.

20. Weizman L, Dayan L, Brill S, Nahman-Averbuch H, Hendler T, Jacob G, et al. Cannabis analgesia in chronic neuropathic pain is associated with altered brain connectivity. Neurology. 2018;91(14):e1285-94.

21. Lichtman AH, Lux EA, McQuade R, Rossetti S, Sanchez R, Sun W, et al. Results of a double-blind, randomized, placebo-controlled study of na- biximols oromucosal spray as an adjunctive therapy in advanced cancer patients with chronic uncontrolled pain. J Pain Symptom Manage. 2018;55(2):179-88.e1.

22. van de Donk T, Niesters M, Kowal MA, Olofsen E, Dahan A, van Velzen $M$. An experimental randomized study on the analgesic effects of pharmaceutical-grade cannabis in chronic pain patients with fibromyalgia. Pain. 2019;160(4):860-9.

23. Bellnier T, Brown GW, Ortega TR. Preliminary evaluation of the efficacy, safety, and costs associated with the treatment of chronic pain with medical cannabis. Ment Health Clin. 2018;8(3):110-5.

24. Fanelli G, De Carolis G, Leonardi C, Longobardi A, Sarli E, Allegri M, et al. Cannabis and intractable chronic pain: an explorative retrospective analysis of Italian cohort of 614 patients. J Pain Res. 2017;10:1217-24.

25. Crowley K, de Vries ST, Moreno-Sanz G. Self-reported effectiveness and safety of Trokie ${ }^{\circledR}$ lozenges: a standardized formulation for the buccal delivery of cannabis extracts. Front Neurosci. 2018;12:564.

26. Finnerup NB, Attal N, Haroutounian S, McNicol E, Baron R, Dworkin RH, et al. Pharmacotherapy for neuropathic pain in adults: systematic review, meta-analysis and updated NeuPSIG recommendations. Lancet Neurol. 2015;14(2):162-73.

\section{CONFLITO DE INTERESSES}

Os autores declaram não possuir quaisquer conflitos de interesse.

\section{FINANCIAMENTO DO ESTUDO}

Os autores declaram que o estudo não teve qualquer financiamento.

\section{ENDEREÇO PARA CORRESPONDÊNCIA}

Ricardo Garcia Silva

E-mail: rgsilva21@hotmail.com

https://orcid.org/0000-0002-9766-6727

Recebido em 17-12-2019

Aceite para publicação em 01-06-2020 


\section{ABSTRACT}

\section{CANNABINOIDS IN CHRONIC PAIN: AN EVIDENCE-BASED REVIEW}

Aim: To review the current evidence regarding the effectiveness of cannabinoids in the treatment of chronic pain.

Data sources: MEDLINE/PubMed, Cochrane Library, TRIP Database, National Guideline Clearing House, Canadian Medical Association Practice Guidelines.

Methods: In August 2019, we searched for meta-analysis (MA), systematic reviews (SR), observational studies (OS), clinical trials (CT), and guidelines, published in Portuguese and English, with no time limit, using the MeSH terms 'cannabis' and 'chronic pain'. The studies that were included were the ones regarding cannabinoid treatment for chronic pain of any cause in adults. Any study regarding acute pain was excluded. The Strength of Recommendation Taxonomy scale, from the American Academy of Family Physicians, was applied to assign levels of evidence (LE) and strength of recommendation (SOR).

Results: A total of 244 articles were obtained, and 16 of those fulfilled the inclusion criteria: nine SR, four randomized doubleblind CT, two retrospective case-series studies, and one prospective case-series study. All studies focused on chronic pain, but the etiology varied between studies (oncologic pain, neuropathic pain, rheumatologic pain, visceral pain). The results were not consistent between the studies. Although there may be some benefits to neuropathic pain, the reviews are all in agreement that greater dimension and longer duration CT are necessary for cannabinoids usage to have robust evidence. Gastrointestinal as well as motor and cognitive function adverse effects may occur, especially with preparations containing a higher dosage of tetrahydrocannabinol. There is no evidence for treatments with cannabinoids in rheumatologic pain. LE 1 was not assigned to any of the included studies.

Conclusions: The therapeutic usage of cannabinoids, while promising and with likely benefit identified in some small studies for some types of chronic pain (especially neuropathic), has limited evidence (SOR B), and requires some higher quality, bigger studies. The possible long-term efficacy and side effects should be considered on longer clinical trials, which may be achieved with the rise of the prescriptions of these compounds. Regarding the current evidence, cannabinoids can be the last resort solution in cases of refractory neuropathic and oncologic pain.

Keywords: Cannabis; Cannabinoids; Chronic pain. 